\title{
A PERCEPÇÃO DE SAÚDE PARA ADOLESCENTES OBESOS
}

\author{
THE PERCEPTION OF HEALTH TO OVERWEIGHT ADOLESCENTS \\ COMO LOS ADOLESCENTES OBESOS PERCIBEN EL CONCEPTO DE \\ SALUD
}

\author{
Maria das Graças Carvalho Ferriani ${ }^{1}$ \\ Stefanie Dechen ${ }^{2}$ \\ Taisa Silva Dias ${ }^{3}$ \\ Marta Angélica lossi ${ }^{4}$
}

RESUMO: O presente estudo tem como objetivo conhecer a percepção de saúde para adolescentes obesos que participam do Programa Multidiciplinar de Assistência ao Adolescente Obeso, desenvolvido no Campus de Ribeirão Preto - USP. Foi utilizada a abordagem qualitativa fundamentada em Minayo (1996); os dados foram coletados através de frases e redaçōes de 17 adolescentes integrantes do programa. A análise e interpretação dos dados foi ancorada na técnica de análise temática proposta por Bardin (1977), onde identificou-se os seguintes núcleos de sentido: saúde é beleza, alimentação e higiene e prática de esportes. Assim, o estudo evidenciou que a imagem corporal ideal que o adolescente busca para si, está relacionada com os padrōes de beleza ditados pela midia e com estereótipos de perfeiçāo fisica. Outro aspecto detectado no estudo, é que os adolescentes têm consciência da importância de praticar esportes e ter uma alimentaçäo balanceada.

PALAVRAS-CHAVE: adolescência, obesidade, saúde

\section{INTRODUÇÃO}

$\mathrm{Na}$ adolescência o ser humano sofre transformações aceleradas em seu processo de crescimento e desenvolvimento. Segundo a literatura, é o periodo em que as transformaçōes fisicas-biológicas da puberdade associam-se àquelas de âmbito psicossocial, delas resultando a realização do jovem e posteriormente do adulto.

Para Chipkevitch (1987), năo se pode falar da adolescência sem falar do corpo e do corpo sem falar da mente. Assim, as intensas transformações fisicas e biológicas desta idade, influenciam todo o processo psicossocial da formação da identidade do adolescente.

Por outro lado, a obesidade em crianças e adolescentes vem crescendo de forma assustadora em nossa sociedade. Segundo a Organização Mundial da Saúde, o mundo vive uma epidemia de obesidade, trazendo riscos à saúde e preocupando pesquisadores. Os paises desenvolvidos têm concentrado seus esforços para a reduçăo da obesidade, bem como para modificação do padrão alimentar e redução do sedentarismo (COUNCIL, 1989). Um desses grupos é o de adolescentes que quando obesos, apresentam maiores probabilidades de se tornarem adultos obesos (Malina; Bouchard, 1991).

Atualmente, observa-se em nosso país, uma transiçăo epidemiológica marcada pela coexistência de desnutriçăo energético-proteica e obesidade.

\footnotetext{
'Orientadora. Prof'. Titular do Dep. de Enferm. Matemo Infantile Saúde Pública-EERP/USP.

${ }^{2}$ Bolsista do PIBIC/CNPq.

${ }^{3}$ Bolsista CNPq, graduanda em emfermagem.

${ }^{4}$ Enfermeira do PROASE e doutoranda em Enfermagem EERPIUSP.
} 
Para Mannarino e Gerude (1993), a obesidade também no Brasil passou a fazer parte da agenda de Saúde Pública, pois está associada à vários fatores de risco, aumentando a ocorrência de doenças crônico-degenerativas, dentre outras.

A obesidade pode ter inicio em qualquer época da vida, principalmente nos períodos de aceleração do crescimento. A adolescência sendo um periodo caracterizado por intensas transformaçōes fisicas e psicológicas conforme mencionamos, é considerado um periodo oportuno para o tratamento e prevenção da obesidade (Santos, 1998).

O adolescente obeso enfrenta dificuldades em aceitar seu próprio corpo e o fato de ser obeso. Considerando todos estes aspectos, interessa-nos ter como objeto de estudo nesta pesquisa, o adolescente obeso.

\section{OBJETIVO}

Conhecer a percepção de saúde de adolescentes obesos, oriundos das escolas de do ensino fundamental da rede pública, junto ao Programa de Assistência Primária à Saúde Escolar - PROASE - e que participam de um Programa Multidisciplinar de Assistência ao Adolescente Obeso, que vem sendo desenvolvido no Campus de Ribeirăo Preto - USP.

\section{PERCURSO METODOLÓGICO}

Trata-se de um estudo qualitativo, por trabalhar com o universo de significados, motivos, crenças e atitudes, o que corresponde a um espaço mais profundo das relaçőes, dos processos e dos fenômenos que não podem ser reduzidos a operacionalização de variáveis quantitativas (Minayo, 1996).

O Programa Multidisciplinar de Assistência ao Adolescente Obeso, iniciou suas atividades em outubro de 1998 no Campus de Ribeirão Preto-USP, junto a 30 adolescentes de ambos os sexos, na faixa etária de 10 a 14 anos. Estes adolescentes săo oriundos do sistema público de ensino fundamental da cidade de Ribeirão Preto, que possuem o PROASE. As enfermeiras do PROASE foram as responsáveis por detectar e indicar os adolescentes que se apresentavam ao menos com sobrepeso, ou seja, com Índice de Massa Corpórea (IMC) de no minimo 25kg/ $\mathrm{m}^{2}$. Antes de iniciarem as atividades do programa, esses adolescentes também foram submetidos a uma avaliação médica.

A equipe multidisciplinar que integra este programa é constituida por professores de educação fisica, médico, nutricionistas, psicólogos e enfermeiras; além de alunos de graduação e pós-graduação na área de enfermagem e psicologia. As atividades desenvolvidas por essa equipe e oferecidas aos adolescentes săo: atividades motoras adaptadas, educação nutricional, ( com ênfase nas mudanças de comportamento e reeducaçăo alimentar) e orientação psicológica em grupo. Estas atividades ocorrem nas dependências do Centro de Educação Fisica, Esportes e Recreação - CEFER, Campus de Ribeirão Preto - USP.

A equipe de enfermagem desenvolve reuniōes quinzenais com esse grupo de adolescentes, por um periodo de 1 hora, na Escola de Enfermagem de Ribeirão Preto-EERP - USP, onde eles têm a oportunidade de discutir e refletir questões referentes a adolescência como um todo. Partindo das modificações fisicas-biológicas que ocorrem, abordando a sexualidade, namoro, familia, mudanças do pensamento, comportamento; enfim, questões pertinentes que fazem parte do quotidiano desses adolescentes.

Já nos primeiros encontros, após ser colocado para os adolescentes o que viria a ser o programa, foi questionado o porquê de eles quererem fazer parte do programa. Notamos que a palavra saúde estava muitas vezes presente em suas falas. Assim, surgiu o interesse de estarmos conhecendo a percepção desses adolescentes obesos sobre o conceito de saúde. Afinal, o que é ter saúde para esses adolescentes? 
A coleta de dados ocorreu durante os nossos encontros, após o consentimento dos responsáveis (pais dos adolescentes), seguindo as normas da Resolução n 196/96-CNS.

O material utilizado e analisado para obtermos esse conhecimento, foram as redaçöes e frases de 17 adolescentes, (esses adolescentes são os que estavam presentes em uma das reuniões) sobre o tema: "O que é ter saúde?". Para complementar os dados, utilizamos a observaçăo livre, que foi realizada durante as reuniões.

\section{ANÁLISE DOS DADOS}

Para análise e interpretação dos dados desta pesquisa, ancoramo-nos na técnica de análise temática proposta por Bardin (1977).

Segundo Minayo (1996), a análise temática consiste em descobrir os núcleos de sentido que compőem uma comunicação cuja presença signifique alguma coisa para o objetivo analítico visado.

De acordo com Gomes (1994,p.74), além de se buscar respostas para as questōes, com essa técnica pode-se caminhar na direção da "descoberta do que está por trás dos conteúdos manifestados, indo além das aparências do que está sendo analisado".

\section{CARACTERIZAÇĀO DOS ATORES SOCIAIS} temáticos:

De acordo com a análise dos dados coletados apreendemos os seguintes núcleos

Os 17 adolescentes que participaram da pesquisa encontram-se na faixa etária de 9 a 13 anos de idade, sendo 12 do sexo masculino e 05 do sexo feminino, o que vem ao encontro com a literatura estudada, ou seja, o percentual de meninos obesos é maior que o de meninas, de acordo com Fonseca et al. (1998). A escolaridade dos adolescentes está entre $2^{\mathrm{a}}$ e $6^{\mathrm{a}}$ série do $1^{\circ} \mathrm{grau}$ das escolas públicas do ensino fundamental do Municipio de Ribeirăo Preto, próximas ao Campus de Ribeirăo Preto, onde se desenvolve o Programa Multidisciplinar de Assistência ao Adolescente Obeso. A faixa etária das mães dos adolescentes está entre 29 e 48 anos de idade, e os pais entre 34 e 44 anos de idade. A maioria dos pais possue, apenas, o ensino fundamental incompleto, exceto 02 pais e 03 mães que possuem ensino fundamental, e 01 pai que possui ensino médio completo.

Observa-se que a maioria das familias tem rendimentos mensais entre 10 a 15 salários mínimos, sendo que duas delas está entre 6 a 15 , uma entre 3 a 5 , duas com até 3 e uma entre 15 a 20 salários mínimos. Em média, o número de pessoas nas familias está entre 05 a 06 , exceto 3 familias constituidas por 04 pessoas e outra por 03.

Cabe ressaltar que 07 adolescentes possuem avós com história de Hipertensão Arterial, além dos pais de um destes adolescentes. Em relação à obesidade, dentre os 17 adolescentes sujeitos do estudo, foram identificados: 06 avós paternos, 04 avós maternos, 06 pais, 08 mães e 01 irmão obesos. No que se refere a Diabete Mellitus, 03 adolescentes possuem avós com história de diabetes. Além do que, 06 adolescentes possuem 02 avós paternos e 01 avô materno com história de infarto do miocárdio.

\section{SAÚDEÉ BELEZA}

A análise dos conteúdos das falas dos adolescentes revelou uma temática central "saúde é beleza" - em torno da qual podemos sintetizar as preocupaçōes desses sujeitos sociais acerca do que é ter saúde. Esse sentido nos foi revelado não só a partir dos textos, figuras, como também no momento das reuniōes e discussões com o grupo em relação a temática. Isso nos possibilitou conhecer as idéias, mitos, valores, a fantasia e o conhecimento 
sobre o que é ter saúde. Entre as falas abaixo, podemos evidenciar:

Uma mulher bonita é ter saúde.

É quase igual saúde, mas beleza é você cuidar do corpo.

A Tiazinha é uma mulher bonita, gostosa. A Sheila Carvalho é uma mulher gostosa, saudável e bonita.

Ser bonita é ter boa saúde, estar bem com a vida.

Nestas falas, podemos verificar que ter saúde é ter um corpo bonito. Este fato é compreensível, uma vez que a fase em que se encontram (adolescência), os padrões de beleza ditados pela mídia e de estereótipos de perfeição fisica que o adolescente busca para si, numa fase da vida de intensas transformaçōes biopsicossociais que estão vivenciando, o que acaba por gerar angústia, insegurança, quando o assunto é o corpo (Suplicy, 1995).

\section{PRÁTICA DE ESPORTES}

Outro aspecto considerado pelos adolescentes para ter saúde e ser bonito é praticar esportes (nadar, dançar, andar de patins, voleibol, handebol). As figuras desenhadas de corpos colocados na folha da redação foram desenhos de atrizes mostrando o corpo, de crianças andando de bicicletas, correndo, nadando.

As falas abaixo explicitam o interesse pelo corpo e a consciência que é necessário cuidar do mesmo, conforme segue:

Ter saúde é preciso praticar muito esporte.

A saúde depende de nós fazendo exercicio.

Fazer atividades tipo futebol, basquete.

Saúde é fazer esporte saudável e que você gosta.

Observa-se que há uma conscientizaçăo dos adolescentes em relação a importância da prática de esportes. A atividade fisica é um importante determinante das caracteristicas físicas do adolescente. Pudemos constatar, através das falas dos adolescentes nas reuniōes quinzenais, que estes, antes de ingressarem no Programa, não praticavam nenhum tipo de exercicio fisico. A maioria dos adolescentes participantes da pesquisa, permaneciam em média de 2 a 4 horas em frente à televisăo, alimentando-se com sanduiches, bolachas recheadas, pipoca, refrigerantes, etc. No decorrer das reuniōes com o grupo de vivência, os adolescentes explicitaram que o tempo que permaneciam em frente à televisão, atualmente, é utilizado para dedicar-se às atividades do programa.

Santos (1998) afirma que é no corpo "obeso" que o adolescente vivencia o sentido de seu existir, e que as manifestaçōes do existir destes adolescentes "obesos", refletem uma época na qual há a valorização do corpo "magro". O adolescente mantém constante preocupação com o peso, visando um ideal de beleza imposto pelo corpo magro, e a não aceitação de seu corpo o leva a se sentir marginalizado na sociedade, o que dificulta suas relações interpessoais.

A autora conclui, que apesar da percepção do corpo "obeso" pelos adolescentes continuar sendo-ao-mundo, e a experiência pré-reflexiva de seu corpo a direcionar para uma açäo que é a busca de dietas ou tratamentos inspirados no outro e na necessidade de estabelecer relacionamentos afetivos com meninos(as); e enfim, libertarse.

De acordo com alguns autores como Dietz (1993), a obesidade em adolescentes resulta do desequilibrio entre atividade reduzida e excesso de consumo de alimentos calóricos, tendo mostrado que o número de horas que um adolescente passa assistindo televisão é importante 
fator associado à obesidade, acarretando um número de $2 \%$ na prevalência da obesidade para cada hora adicional de televisăo em jovens de 12 a 17 anos.

\section{ALIMENTAÇÄO E HIGIENE}

$\mathrm{Na}$ fala dos adolescentes, observa-se que a saúde também está associada a uma alimentação balanceada e a higiene. Embora o Programa Multidisciplinar de Assistência ao Adolescente Obeso, implantado no Campus - USP, não seja objeto nesta pesquisa de avaliação, podemos inferir que este vem contribuindo, sobremaneira, na conscientização destes adolescentes em relação aos hábitos alimentares, conforme podemos evidenciar nas falas abaixo:

A saúde de nosso corpo depende basicamente da nossa alimentação.

O corpo precisa de energia para que todos os organismos possam funcionar. Essa alimentação, que deve ser variada.

Os sais minerais são necessários para a formação dos ossos, dentes e do sangue.

Saúde é não comer um pacote cheio de chocolate nescau.

Ter saúde é não ter gordura no sangue. Comer verduras, dividir os alimentos certo.

Ter saúde não é comer 1 pacote de bolacha.

Ter uma comida saudável e balanceada, mas que mate a fome.

Estas falas são contrárias ao que vivenciamos no quotidiano e que a literatura chama atenção, em que o hábito de omitir refeições, especialmente o desjejum, o consumo de refeições rápidas fazem parte do estilo de vida dos adolescentes, sendo considerados comportamentos importantes que podem contribuir para o desenvolvimento da obesidade. O consumo alimentar como um todo năo tem sido consistentemente associado ao estado nutricional.

De acordo com as nossas observaçōes durante as reuniões do grupo de vivência, os adolescentes manifestaram que, antes de participarem do programa, eles tinham o hábito de comer rápido e compulsivamente, além de mastigarem pouco. Percebe-se, portanto, que estes adolescentes de alguma forma estão recebendo uma educação alimentar e procurando mudar seus comportamentos.

Segundo Klesges (1988), outra explicaçăo provável é que fatores da dieta são mais importantes no inicio da obesidade, ao passo que a atividade fisica e/ou fatores metabólicos têm importância na manutençăo da obesidade.

O adolescente percebe a necessidade de auto-controle, mudanças de hábitos alimentares e atividades físicas através de dietas e tratamentos (Santos,1998).

A prevençăo da obesidade, assim como a reduçăo de peso excessivo, passa necessariamente por duas possibilidades: aumentar o gasto energético e reduzir o consumo. A literatura, com frequeência, aponta para a importância de se iniciar precocemente durante 0 desenvolvimento dos individuos, consumo alimentar e atividade física adequados. Com isso, haverá maior chance de reduzir a prevalência de obesidade, seja nos adolescentes, seja entre os adultos. A prevenção da obesidade depende da promoçăo de uma vida saudável, mesmo porque comportamentos como realizar atividade fisica, de lazer e comer mais frutas e verduras, agrupam-se nos mesmos individuos (NESTLÉ, 1996).

Embora a obesidade seja um problema importante a ser prevenido já na adolescência, deve-se pensar em políticas de saúde coletiva para os adolescentes, e que essas politicas não reforcem práticas já existentes de comportamentos pouco saudáveis de manutenção de peso e que promovam o acúmulo de atividades fisicas, criando condiçōes objetivas para sua realização. Assim, essas seriam provavelmente, as principais açōes de politicas que visem uma vida saudável para os adolescentes. 


\section{CONSIDERAÇÕES GERAIS}

Retornando ao nosso objetivo, que é conhecer a percepção de saúde de adolescentes obesos, constatou-se que estes consideram que "Ter Saúde" é ser bonito. Outro aspecto apresentado, é ter uma alimentação correta e balanceada, higiene e praticar esportes. Acrescese ainda, os padrões de beleza ditados pela mídia e de estereótipos de perfeição física que o adolescente busca para si, numa fase da vida de intensas transformaçōes biopsicossociais.

Outro aspecto detectado na pesquisa, é que esses adolescentes têm consciência da importância de praticar esportes, ter uma alimentação correta, donde podemos inferir que o Programa Multidisciplinar de Assistência ao Adolescente Obeso, desenvolvido no CampusUSP de Ribeirão Preto, vem contribuindo de forma positiva na conscientização desses adolescentes em relação aos riscos de ser obeso.

É importante ressaltar, que embora tenha-se constatado na caracterizaçăo dos atores sociais, pais e avós apresentando história de Hipertensão Arterial, Diabetes Mellitus, Obesidade e problemas cardiacos, e este não ser o objetivo deste estudo, será necessário elaboração de novas pesquisas objetivando aprofundar a temática.

ABSTRACT: This work aims at learning about the perception of health of overweight adolescents participating in the Multidisciplinary Program of Assistance to Overweight Adolescents developed in the Campus of Ribeirão Preto (University of São Paulo). The qualitative approach based on Minayo (1994) was adopted. Data were collected from sentences and compositions done by 17 adolescents, who were participating in the program. The theme of the compositions and sentences were: "What being healthy means?". Data analysis and interpretation was done based on the thematic analysis technique proposed by Bardin (1976). Through this analysis, the following meaning units were identified: "health is beauty", "diet and hygiene" and "practice of sports". Therefore, the study showed that the ideal body image adolescents want for themselves is related to the beauty standards established by the media and to the stereotypes of physical perfection. Another aspect detected by the study was that adolescents are aware of the need of practicing sports and having a balanced diet.

KEYWORDS: adolescence, overweight, health

RESUMEN: El presente estudio tiene como objetivo conocer la percepción de salud entre los adolescentes obesos que participan del Programa Multidisciplinar de Asistencia al Adolescente Obeso, desarrollado en el Campus de la Universidad de São Paulo en Ribeirão Preto. Se utiliza la investigación de tipo cualitativo, que está fundamentada en Minayo (1994); los datos se recogen a través de las frases y redacciones de 17 adolescentes que han participado en el programa sobre el tema "Qué significa tener salud". El análisis y la interpretación de los datos se han basado en la técnica de análisis temático propuesta por Bardin (1976), con la identificación de los siguientes núcleos de sentido: "salud es belleza", "alimentación e higiene" y "práctica de deportes". Asi, el estudio ha evidenciado que la imagen corporal ideal que el adolescente busca está relacionada con los patrones de belleza dictados por la midia y con estereotipos de perfección física. Otro aspecto que se detecta en el estudio es que los adolescentes tienen conciencia de la obesidad.

PALABRAS CLAVE: adolescencia, obesidad, salud

\section{REFERÊNCIAS BIBLIOGRÁFICAS}

BARDIN, L. Análise de conteúdo. Lisboa: Edições 70, 1977. 
CHIPKEVITCH. E.; O adolescente e o corpo. Pediatria Moderna, v. 23, n.6, p. 231-237, jul./ago., 1987.

DIETZ,W.H. Factors increasing risk of obesity and potencial for prevention overweight in childhood. In: Workshop in Prevention of Obesity Population at Risk, Etiologic Factors and Intervention Strategies, Baltimore, National Institutes of Health/ National Institute of Diabetes and Digestive and Kidney Diseases, p.64, 1993.

FONSECA,MV.M.; SICHIERI,R.; VEIGA, G.V. Fatores associados à obesidade em adolescentes, Rev. Saúde Pública, v. 32, n. 6, p. 541-9,1998.

GOMES, R. A análise de dados em pesquisa qualitativa, p. 67-80. In: MINAYO, M.C.M. (Org.) Pesquisa Social: Teoria, Método e Criatividade. Petrópolis: Editora Vozes, 1994.

KLESGES, R.C. et al. Accuracy of self-reports of food intake in obese and normal weight individuals: effects of parental obesity on reports of children's dietary intake. Am. J. Clin. Nutr., v. 48, p. 1252-6, 1988.

MALINA, R.M.; BOUCHARD,C. Growth, maturation and physical activity. New York, Versa Press, 1991.

MANNARINO,I.C.; GERUDE, M. Obesidade. In: MANNARINO, I.C.; GERUDE, M.. Terapia Nutricional. Sāo Paulo: Sarvier, 1993. p. 115-6.

MINAYO, M.C.S. O desafio do conhecimento: pesquisa qualitativa em saúde. 4. ed. São Paulo: Hucitic/ ABRASCO, 1996.

National reserch COUNCIL. Diet and health: implication for reducing chronic diseases risk. Committee on Diet and Health. Washington, DC: Academy Press, p. 231-237, jul./ago., 1987.

NESTLÉ, M. Fruits and Vegetables: protective or just fellow travelers. Nutr. Rev., v. 54, n. 8, p. 255-257. 1996.

SANTOS, M.F. O sentido de existir de adolescentes que se percebem obesas: uma abordagem à luz de Merleau- Ponty. 1998. 210p. Tese (Doutorado) - Escola de Enfermagem de Ribeirão Preto, Universidade de São Paulo, Ribeirāo Preto.

SUPLICY, M. Sexo se aprende na escola. Säo Paulo: Olho d' Água, 1995. p. 120.

Recebido em dezembro de 2000

Aprovado em maio de 2001 